\title{
Pierre Salama
}

\section{Eine neue Art von Finanzkrisen in Lateinamerika}

\section{Zwei Typen von Krisen}

Die lateinamerikanischen Finanzkrisen der 1980er Jahre unterscheiden sich grundlegend von denen der 1990er Jahre. Die früheren resultierten aus der ungeheuren Belastung durch die Außenverschuldung und aus der Notwendigkeit einen großen Teil der internen Ressourcen für den Schuldendienst einzusetzen. Die neueren Krisen beruhen dagegen auf extrem starken finanziellen Zwängen, die von außen kommen.

Kehren wir kurz zu den früheren Finanzkrisen zurück: Anfang der 1980er Jahre sah sich Mexiko außerstande, seine Schulden zurückzuzahlen, worauf die internationalen Finanzmärkte auf extrem brutale Weise reagierten: Die lateinamerikanischen wie auch mehrere asiatische Länder erhielten internationale Kredite nur noch unter der Bedingung, dass sie beim Internationalen Währungsfonds (IWF) einen letter of intent unterschrieben. Von nun an mussten sie einen erheblichen Teil ihres Schuldendienstes über ihre internen Ressourcen finanzieren und die Bedingungen der Umschuldung des anderen, wesentlicheren Teils mit dem IWF aushandelten. Sie mussten sich zu einer Austeritätspolitik verpflichten, die eine drastische Reduzierung der öffentlichen Ausgaben und eine maximale Abwertung ihrer Währungen einschloss, um ihre Exporte zu steigern und einen positiven Saldo ihrer Handelbilanz zu erreichen, so dass ausreichend Devisen zur Verfügung stehen würden, um einen Teil ihres Schuldendienstes zu erfüllen. Insgesamt führte dies dazu, dass in einigen Ländern die Exporte die Importe um rund 30\% überstiegen und die Rückzahlung der Auslandsschuld zwischen 2 und 5\% des gesamten Bruttoinlandsproduktes ausmachte - das ist mehr als die Siegermächte nach dem Ersten Weltkrieg im Vertrag von Versailles von Deutschland als Reparationszahlungen auferlegten. Die Nettoströme des Kapitals kehrten sich auf diese Art um: Lateinamerika exportiert nun mehr Kapital als es bekommt. Der Preis dafür ist extrem hoch: eine zehnjährige Depression, die vor allem auf die Einschnitte bei den öffentlichen Ausgaben zurückgeht, in der Folge eine zurückgehende Investitionsneigung, in mehreren Ländern als Effekt der massiven Abwertungen eine Hyperinflation, ein Anwachsen der Armut und der sozialen Ungleichheiten. In dieser Situation leiden die Armen unter der Inflation um 
so mehr, je ärmer sie sind. Die Ökonomen bezeichnen dieses Phänomen als "Inflationssteuer“. Der auswärtige Schuldendienst stellt eine Ausgabe des öffentlichen Haushalts dar, da ein großer Teil der Schulden aus öffentlichen Kreditaufnahmen besteht, und da sich die Regierungen meist dafür entschieden haben, das Wechselkursrisiko der privaten Verschuldung zu übernehmen. Die öffentlichen Einnahmen blieben unzureichend, gleichzeitig bestand die Verpflichtung, das Budgetdefizit zu reduzieren, aber die mit dem Schuldendienst zusammenhängenden Ausgaben stiegen um so mehr an, je stärker die Währung abgewertet wurde. Daraufhin gaben die Regierungen Schatzbriefe aus, die sie dadurch attraktiv machten, dass sie ihren Wert entweder mit einem Inflationsausgleich oder mit einer Angleichung an den inoffiziellen Wechselkurs, der als Indikator für zukünftige Geldentwertung gilt, indexierte. Dieser stark inflationistische Kontext und die durch den Schwarzmarktkurs angekündigten Abwertungen erlaubten somit den wohlhabendsten 5\% der jeweiligen Bevölkerung, sich mit Hilfe dieser indexierten Produkte weiter zu bereichern. Die Auslandsschuld generierte eine Inlandsschuld, die schwer zu kontrollieren ist und die sich für die Banken wie für einen kleinen Teil der Bevölkerung als immer lukrativer erwies. Demgegenüber waren die Auswirkungen des Schuldendienstes für die große Mehrheit der Bevölkerung, und da vor allem für die unteren Schichten, katastrophal. Indirekt waren es die Armen, die nicht über Dollarguthaben verfügten, aber für die Bezahlung der Auslandschuld aufkamen - eine Auslandsschuld, die in einigen Ländern (Argentinien, Venezuela, teilweise auch Mexiko) ursprünglich dadurch entstanden war, dass die Reichen ihre Kapitalien in die USA transferiert hatten.

Die Krisen der 1990er Jahre sind jedoch anderer Art. Der Ausweg aus der vorhergehenden Krise geschah durch eine plötzliche und brutale Liberalisierung der Gesamtheit der Märkte. Die Inflation wurde gestoppt, es kam wieder zu Wachstum. In den ersten Jahren ging auch die Armut zurück, gelegentlich, wie in Brasilien, sogar in erheblichem Ausmaß. Sehr bald traten aber Finanzprobleme auf, die kaum überwindbar erschienen. Die Wirtschaft funktionierte mehr und mehr in der Art des „Kasinokapitalismus“, wie Keynes ihn bezeichnete. Die Fortsetzung des Wachstumskurses stieß in zunehmendem Maß auf finanzielle Hindernisse. Die Raten des Wachstums schwächten sich ab und schwankten immer stärker.

Besonders große Schwankungen gab es in Mexiko und in Argentinien mit einer Krise im Jahr 1995, dann 1998 einer in Brasilien und im folgenden Jahr wiederum einer in Argentinien, wo es 2002 zu einem massiven Einbruch des Sozialprodukts von fast $11 \% \mathrm{kam}$, was dazu führte, dass mehr als die Hälfte der Bevölkerung unter der Armutsgrenze lebte, während Mexiko, Brasilien und die meisten lateinamerikanischen Länder 2002 und 2003 ein stark verlangsamtes Wachstum erlebten. Dennoch waren die Schwankungen der 1990er Jahre weniger ausgeprägt als diejenigen der 1980er Jahre, die als „verlorenes Jahr- 
zehnt" bezeichnet werden. Auch die Ursachen dieser Schwarkungen unterscheiden sich - damals lagen sie im Schuldendienst, der auf Kosten der nationalen Ressourcen ging, im letzten Jahrzehnt dagegen in der gesteigerten finanziellen Abhängigkeit als Folge der neuen Wachstumsmuster, die zur Überwindung der Hyperinflationskrisen implantiert wurden.

Tabelle 1: Wachstumsraten des BIP in den wichtigsten lateinamerikanischen Ländern

\begin{tabular}{|l|c|l|l|l|l|}
\hline & Argentinien & Brasilien & Chile & Kolumbien & Mexiko \\
\hline 1991 & 10,6 & 1,0 & 7,3 & 1,8 & 4,2 \\
\hline 1992 & 9,6 & $-0,3$ & 10,9 & 3,6 & 3,7 \\
\hline 1993 & 5,9 & 4,5 & 6,6 & 4,4 & 1,8 \\
\hline 1994 & 5,8 & 6,2 & 5,1 & 5,9 & 4,4 \\
\hline 1995 & 2,9 & 4,2 & 9,0 & 4,9 & $-6,1$ \\
\hline 1996 & 5,5 & 2,5 & 6,9 & 1,9 & 5,4 \\
\hline 1997 & 8,0 & 3,1 & 6,8 & 3,3 & 6,8 \\
\hline 1998 & 3,8 & 0,1 & 3,6 & 0,8 & 5,1 \\
\hline 1999 & $-3,4$ & 0,7 & $-0,1$ & $-3,8$ & 3,7 \\
\hline 2000 & 0,8 & 4,4 & 4,4 & 2,7 & 6,6 \\
\hline 2001 & $-4,4$ & 1,5 & 2,8 & 1,4 & $-0,4$ \\
\hline 2002 & $-10,8$ & 1,5 & 2,1 & 1,2 & 0,9 \\
\hline $2003^{\circ}$ (geschätzt) & 5,0 & 1,8 & 3,5 & 2,0 & 2,4 \\
\hline
\end{tabular}

Quelle: Cepal: Proyecciones de America Latina y Caraibes 2003

Es gab nicht nur häufige, sondern auch sehr heftige Konjunkturschwankungen, deren Ausprägungen eher an die europäischen Zyklen des 19. Jahrhunderts mit ihren starken Ausschlägen nach oben und unten erinnerten, als an die moderaten Bewegungen des 20. Jahrhunderts. Dieser häufige Wechsel traf die Armen sehr viel mehr als das geringe Wachstum.

\section{Die Auswirkungen der Liberalisierung}

Ein kurzer Rückblick auf den internationalen Kontext zeigt, dass Bankkredite für den internationalen Zahlungsverkehr der lateinamerikanischen Ökonomien zur Restgröße wurden, sie dienten in erster Linie zur Refinanzierung der Schulden, wie sie, entsprechend den letters of intent, mit dem IWF vereinbart wurden (daher auch die Bezeichnung „unfreiwillige Kredite"). Dagegen nahmen Anleihen, Schatzanweisungen und Aktien, die allesamt Finanzinvestitionen darstellen, einen wichtigen Platz ein. Auch ausländische Direktinvestitionen, die in den 1980er Jahren marginal geblieben waren, erlebten in der Mitte der 1990er Jahre einen ungewöhnlichen Aufschwung.

Im nationalen Kontext ist demgegenüber festzustellen, dass die Realsphäre der Ökonomie in einzelnen Ländern mehr oder weniger von der Krise der 1980er Jahre in Mitleidenschaft gezogen wurde. Die brutale Öffnung der Märkte 
führte in einigen Ökonomien zu erheblichen Defiziten der Handelsbilanz, welche die bisherigen Überschüsse ersetzte, die ihrerseits Ergebnis des ausgeprägten Protektionismus und der Krise waren. Tatsächlich war die Wettbewerbssituation der meisten Länder so schwach, dass sie nicht in der Lage waren, den massiven Zustrom ausländischer Waren, der mit der plötzlichen Liberalisierung einsetzte, zu verhindern, nicht nur weil eine „Entwaffnung" bei den Einfuhrzöllen stattgefunden hatte, sondern auch eine erhebliche Reduzierung der Subventionen für den Exportsektor, und man ganz allgemein zu einer Politik der Zinserhöhungen übergegangen war. Ein weiterer Faktor, der das Defizit stark ansteigen ließ, lag in der realen Aufwertung der Währung. Diese ergab sich automatisch aus der neuen Anti-Inflations-Politik: das Ende der Preissteigerungen führte zur Aufwertung, was Importe begünstigte, aber Exporte erschwerte.

Da aus dem bisherigen Handelsbilanzüberschuss ein Defizit wurde, war der Schuldendienst darüber nicht mehr zu finanzieren. Gleichwohl führten Liberalisierung und Deregulierung sowie eine Hochzinspolitik dazu, dass spekulatives Kapital angezogen wurde. In einer ersten Phase sicherte dieses die Finanzierung des Schuldendienstes und des Defizits dex Handelsbilanz. Diese Wirtschaftspolitik erhöhte die Kreditwürdigkeit bei den internationalen Finanzmärkten, so dass Kapital in die Länder strömte, die Reserven der Zentralbanken zunahmen und die Aufwertung der nationalen Währungen sich weiter fortsetzte, und zwar nominal wie real (dies mit Ausnahme von Argentinien, wo der gesetzlich fixierte nominale Wechselkurs durch den Ausbruch der Finanzkrise Ende des Jahre 2001 praktisch „hinweggefegt“ wurde).

Die erste Krise ereignete sich Mitte der 1990er Jahre in Mexiko. Das Handelsbilanzdefizit schwoll derartig an, dass die ausländischen Investoren daran zu zweifeln begannen, ob das Land die Gesamtheit seiner finanziellen Verpflichtungen erfüllen würde. Aus Angst vor einer Abwertung setzte eine Flucht nationalen wie internationalen Kapitals ein. Die Währung wurde zwei Mal stark abgewertet. Die US-amerikanische Regierung erklärte sich der mexikanischen gegenüber zu massiven Stützungsmaßnahmen bereit, um die dramatische Kapitalflucht zu stoppen. Was man hier erlebte, war eine neue Art von Krisen: erstmalig werden die Auswirkungen einer brutalen und massiven Liberalisierung dieser Ökonomien deutlich sichtbar. Gleichzeitig entstand damit ein Kontext, der auch in anderen schwachen Ländern wie in Argentinien das Abfließen von Kapital begünstigte (der „Tequila-Effekt“).

Die bedenkenlose Öffnung der Kapitalmärkte führt zu extrem harten Folgen für die Realsphäre der Ökonomie: um die Kapitalflucht zu stoppen, mussten astronomisch hohe Zinssätze eingeführt werden, was sehr schnell und ohne die Kapitalabflüsse zu verlangsamen, zu einer Lähmung der Produktion führte 
und die ökonomische Krise beschleunigte. Die Ökonomen entdeckten erneut, dass die Reaktionsgeschwindigkeit der realen Sphäre sehr viel langsamer ist als diejenige der finanziellen Sphäre. So zeigt sich etwa der positive Effekt einer erhöhten Investitionsquote auf das Wachstum erst mit einer gewissen Verzögerung. Selbst wenn es zu einer erheblichen Abwertung der nationalen Währung aufgrund von Spekulationsbewegungen kommt, lässt diese die Exporte erst nach einiger Zeit steigen. Und auch das findet in einer Wirtschaft, die nur zum Teil offen ist und deren Exporte nicht in erster Linie in Rohstoffen bestehen, nur dann statt, wenn die Abwertung wirklich einschneidend ausfällt.

Ungekehrt zeigt sich der Finanzsektor äußerst empfindlich gegenüber spekulativen Bewegungen, und die Geldströme, die plötzlich aus einem Land abfließen, können sehr hoch sein. Diese heftigen und schnellen Reaktionen übertragen sich mit einer starken Hebelwitkung auf die Realsphäre. Hier einige Beispiele: Als die Zinssätze erheblich angehoben wurden (in Brasilien etwa zur Zeit des Höhepunktes der spekulativen Attacken bis zu 50\%), um den Abfluss von Kapital zu stoppen und eine Bewegung in umgekehrter Richtung einzuleiten, zeigten sich als erste Auswirkungen eine massive Verteuerung der Kredite und ein Anstieg der internen Staatsverschuldung, so dass es noch schwieriger wurde, das Staatsdefizit wie versprochen zu reduzieren; es kam sehr rasch zu einer Rezession oder zu einer Verschärfung der Krise in der Realsphäre. Man erlebt hier etwas, was man ein overshooting des Finanzsektors auf den Realsektor nennen könnte. Diese unterschiedlich ausgeprägten Empfindlichkeiten vervielfachen sich, wenn die Wirtschaft relativ geschlossen bleiben, was bei einigen Ländern der Fall ist, auch wenn sie sich in den letzten zehn Jahren immer mehr geöffnet haben. Von mun an ist eine der wichtigsten Quoten, die besondere Aufmerksamkeit verdient, nicht mehr diejenige der Außenschuld im Verhältnis zum BIP, sondern diejenige der Außenschuld im Vergleich zu den Exporten, die in den lateinamerikanischen Ländern extrem geworden ist.

Tabelle 2: Schulden und Exporterlöse

\begin{tabular}{|l|l|l|l|l|}
\hline $\begin{array}{l}\text { Bruttoschuld von 17 Ländem } \\
\text { in Mrd. US Dollar }\end{array}$ & 72000 & 2001 & 2002 & 2003 (geschätzt) \\
\hline In Prozent der Exporte. & 170 & 176 & 715 & 726 \\
\hline
\end{tabular}

Quelle: Dresdner Bank: Latin American Spotlight, März 2003

Zumindest auf der finanziellen Ebene kommt die Krise dann zum Stillstand,

1 Um ein Beispiel zu nennen: während der Diskontsatz Anfang des Jahres 2003 in Brasilien bei $26 \%$ lag, betrug der Zinssatz für Bankkredite 200\%. 
wenn die internationale Glaubwürdigkeit der Regierung wiederhergestellt ist normalerweise als Folge einer starken Abwertung der heimischen Währung, der erheblichen Kürzung der Staatsausgaben mit Ausnahme des Schuldendienstes, der Attraktivität äußerst lukrativer Zinssätze und der finanziellen „Panzerung“, wie sie von den Großbanken und vom IWF als Gegenleistung für eine Fortsetzung des Liberalisierungskurses angeboten wird.

\section{Immer schärfere finanzielle Zwänge, Kasinokapitalismus}

Das Ende der mexikanischen und der argentinischen Krise bedeutete Mitte der 1990er Jahre gleichzeitig den Einstieg in eine neue Phase. Diese hatte zwei wesentliche Kennzeichen: die Salden der Handelsbilanz wiesen in einigen Ländern Überschüsse auf, in anderen nahm zumindest das Defizit ab; die ausländischen Investitionen erlebten einen neuen Aufschwung, insbesondere in Brasilien und in Mexiko, was die Finanzinvestitionen (in Schatzanweisungen und Aktien) auf eine Nebenrolle reduzierte, die dennoch aber nicht gering zu schätzen ist. Sehen wir uns zunächst die Entwicklung der Handelsbilanz an. Die Öffnung der nationalen Ökonomien hat zu einer Veränderung der industriellen Struktur geführt, wobei die Bedeutung der verarbeitenden Industrie insgesamt zugunsten anderer Sektoren wie der Landwirtschaft oder der Ausbeutung von Rohstoffen abgenommen hat. Die industrielle Struktur hat sich durch die Einfuhr von hochmodernen Anlagen geändert. Sie waren durch die Liberalisierung der Austauschbeziehungen und die Aufwertung der eigenen Währung preiswerter geworden, wenngleich diese Entwicklung durch die Abwertungen im Gefolge von Finanzkrisen unterbrochen worden war. Diese Importe bewirkten, dass die Arbeitsprozesse neu organisiert wurden und die Arbeitskräfte flexibler werden mussten, was wiederum - abgesehen von den zeitweiligen Krisen - die Arbeitsproduktivität anwachsen ließ und zu den steigenden Ausfuhren führte. In einigen Ländern verlief die Entwicklung etwas anders, indem komplette Industriezweige verschwanden und das Wachstum der Exporte vor allem auf eine stärkere Spezialisierung auf Rohstoffe aus der Landwirtschaft und aus dem Bergbau zurückgingen, so etwa in Argentinien und Chile. Demgegenüber erhöhten sich in anderen Ländern wie in Mexiko und in mehreren mittelamerikanischen Ländern die ausländischen Investitionen, um entweder für den Binnenmarkt (in Mexiko) oder aber bei geringer Wertschöpfung für ausländische Märkte zu produzieren (Mexiko, Mittelamerika).

Auf unterschiedliche Weise kam es demnach entweder zu bedeutenden Reduzierungen von Handelsbilanzdefiziten oder sogar zu entsprechenden Überschüssen, wie in der nächsten Tabelle am Beispiel von Brasilien zu sehen ist. 
Tabelle 3:Wesentliche Posten der Zahlungsbilanz Brasiliens (in Mrd. US Dollar)

\begin{tabular}{|l|c|c|c|c|c|}
\hline & 1998 & 1999 & 2000 & 2001 & 2002 \\
\hline Handelsbilanz & $-6,6$ & $-1,3$ & $-0,7$ & 2,6 & 9,9 \\
Exporte & 51,1 & 48,0 & 55,1 & 58,2 & 60,4 \\
Importe & 57,7 & 49,3 & 55,8 & 55,6 & 50,5 \\
\hline Dienstleistungsbilanz & $-28,3$ & $-25,8$ & $-25,5$ & $-27,5$ & $-23,2$ \\
davon Zinszahlungen & $-11,40$ & $-14,9$ & $-14,6$ & $-14,9$ & $-13,3$ \\
Dividenden, Gewinne & $-6,9$ & $-4,1$ & $-3,3$ & -5 & $-5,5$ \\
\hline Leistungsbilanz & $-33,5$ & $-25,4$ & $-24,7$ & $-23,2$ & $-7,5$ \\
$\quad$ in Prozent des BIP & 4,25 & 4,81 & 4,15 & 4,58 & 1,62 \\
\hline $\begin{array}{l}\text { Tilgung von mittel- und } \\
\text { langfristigen Schulden }\end{array}$ & $-29,8$ & $-45,4$ & $-32,0$ & $-35,2$ & $-30,0$ \\
\hline Abflüsse insgesamt & $-63,3$ & $-70,8$ & $-56,7$ & $-58,4$ & $-37,5$ \\
\hline Ausländische Direktin- & 28 & 29 & 32,8 & 22,6 & 15,8 \\
vestitionen (ADI) & & & & & \\
$\begin{array}{l}\text { Wertpapieranlagen (An- } \\
\text { leihen und Aktien) }\end{array}$ & -2 & 1,3 & 2,8 & 2,8 & 0,5 \\
\hline $\begin{array}{l}\text { Kurz-, mittel- u. lang- } \\
\text { frist. Auslandsschulden }\end{array}$ & 241,6 & 241,5 & 236,2 & 226,1 & 223,2 \\
\hline
\end{tabular}

Quelle: BACEN

Aber nicht nur die Bedeutung des Schuldendienstes nahm zu, weil dieser absolut anstieg und gleichzeitig die Risikoprämien wuchsen, sondern es trat auch ein neues Element in Erscheinung: Kapitalabflüsse in Form von Dividenden, die aus den Ansprüchen der Aktionäre multinationaler Unternehmen herrühren. In Brasilien stellen sie bereits mehr als ein Drittel des Werts der Schuldzinsen dar. Diese zunehmende Bedeutung des Schuldendienstes resultiert unmittelbar aus dem Aufschwung der ausländischen Investitionen, die vor allem im Bereich der Dienstleistungen stattfinden, bei denen es erhebliche Importe, aber wenig Exporte gibt. Was die ausländischen Investoren anzieht, sind Kapitalanlagen in Zusammenhang mit den wirtschaftlichen Integrationsprozessen in Lateinamerika (vor allem Mercosur und Alena), zum anderen die massiven Privatisierungen in mehreren Ländern (Mexiko, Argentinien). Brasilien stellt dafür ein eindrucksvolles Beispiel dar, da sich hier die Direktinvestitionen in einem Jahrzehnt verzehnfacht haben, so dass das Land inzwischen einen zweimal so hohen Internationalisierungsgrad aufweist wie die USA, wenn man diesen an der Gesamtheit der Aktiva multinationaler Unternehmungen, gewichtet mit dem jeweiligen Sozialprodukt, misst.

Insgesamt waren die Zuflüsse an ausländischen Investitionen nach Lateinamerika im Jahrfünft nach 1995 höher als diejenigen in asiatische Schwellenländer (ohne China), wobei die Unterschiede zwischen den beiden regionalen Blöcken vor allem in diesen Jahren deutlich wurden, sich aber seit Anfang des 
Jahres 2000 wieder verringerten, weil die Kapitalflüsse seit dieser Zeit insgesamt abgenommen haben.

Nun sind noch die Wertpapieranlagen zu betrachten. Diese haben relativ an Bedeutung verloren, blieben aber wichtig. Im wesentlichen sind diese Anlagen per Saldo (Zuflüsse/Abflüsse) zurückgegangen, wobei die Zuflüsse vor allem dazu dienten, frühere Schatzbriefe zu tilgen. Ohne diese Zuflüsse wären die Tilgungen noch viel schwieriger, weshalb man mit hohen Zinssätzen Kapital anzuziehen versucht.

Kapitalabflüsse im Zusammenhang mit der Tilgung der Schulden sowie mit der Zahlung von Zinsen, Dividenden usw. sind unausweichlich, sie sind aber schwierig zu finanzieren. Sie werden teilweise durch den positiven Saldo der Handelsbilanz, sofern ein solcher besteht, aufgebracht, teilweise durch die Devisen, die mit der plötzlichen Flut der Direktinvestitionen in den letzten beiden Jahren hereinkamen, teilweise auch durch Finanzinvestitionen, deren Saldo mittlerweile jedoch gegen Null tendiert, und schließlich zum Teil durch Kredite von internationalen Organisationen. Diese Kredite sind zwar nicht so teuer, zwingen die Länder dafür jedoch auf eine neoliberale Schiene, die ihrerseits in großem Maß für die Instabilitäten und die Verlangsamung des Wachstums verantwortlich ist.

Die Fähigkeit des Wachstumsregimes, das in den 1990er Jahren durchgesetzt wurde, Finanzkrisen zu produzieren, ist bemerkenswert. Der mit ihm verbundene Finanzierungsbedarf ist umfangreich und auf der Ebene von Zinsen, Dividenden und Tilgungszahlungen wenig beherrschbar, auf der Ebene der realen Entwicklung ergibt sich die Schwierigkeit, dass die Exporte gewaltig erhöht werden müssten, um eine dauerhaft positive Handelsbilanz zu erreichen. Die Finanzierungsmöglichkeiten hängen von mehreren Faktoren ab, wovon die einen zu perversen Effekten führen (Liberalisierung der Finanzmärkte und Hochzinspolitik), die anderen durch die nationalen Regierungen schwer kontrollierbar sind (die Handlungsspielräume sind von der jeweiligen Konjunktur abhängig). Insofern wird verständlich, dass das Problem der Glaubwürdigkeit der jeweiligen Regierungspolitik nicht auf einem absoluten, sondern auf einem relativen Niveau besteht. So kann es beispielsweise passieren, dass die Nachfrage nach Kapital zurückgeht, das Angebot aber in noch stärkerem Ausmaß sinkt, so dass eine Lücke entsteht, die von ihrer Größe her bereits ausreicht, um eine Reihe von negativen Wirkungen auszulösen: spekulative Bewegungen, eine Anhebung der Zinssätze, eine Finanzkrise, eine Abwertung der Währung, eine Verlangsamung des Wachstums. In den entwickelten Ländern gibt es seit zwei Jahren einen andauernden Kursverfall an den Börsen, Verluste von groBen Unternehmen, die niedrigere Renditen in Kauf nehmen mussten, und eine Verlangsamung des Wachstums - all dies ist nicht dazu angetan, private Kapitalströme fließen zu lassen.

Das Angebot an Finanzmitteln kann also stärker zurückgehen als die gesunkene Nachfrage, und dies wird insbesondere dann eine Krise herbeiführen, 
wenn eine neue Regierung an die Macht kommt, die bei den Finanzmärkten Zweifel in bezug auf die weitere Wirtschaftspolitik aufkommen lässt. So erging es Lula in Brasilien, bis dieser dem IWF wesentliche Zusicherungen gab, die Politik seines Vorgängers fortzusetzen.

\section{Fazit}

Die Beeinflussung von Zu- und Abflüssen der Zahlungsbilanz erfolgt heutzutage vor allem über die Anpassung der Zinssätze, sie sind ein wesentliches Instrument der Wirtschaftspolitik. Die Anhebung der Zinsen und ihr anhaltend hohes Niveau stellen notwendige, aber nicht ausreichende Bedingungen dar, um Kapital anzuziehen. Allerdings schwächt dies die Banken, da ein Teil ihrer Aktiva entwertet wird (steigende Zinsen führen zu sinkenden Wertpapierkursen), und sie sich außerdem dazu veranlasst sehen, schlechte Kredite zu gewähren. Wenn das Risiko zunimmt, dass Kredite faul werden können, dann verteuert dies insgesamt die Kreditkosten. In der Folge steigt das Budgetdefizit der öffentlichen Hand, selbst wenn Ausgaben gestrichen werden. Investitionsprojekte der Unternehmen werden aufgeschoben, und zwar einerseits wegen ihrer Kosten, andererseits, weil der Kauf von Schatzbriefen rentabler erscheint, als selbst zu investieren. Der Anstieg der Zinsen wirkt konjunkturdämpfend, was einen circulus vitiosus in Gang setzt: Die hohen Zinsen vergrößern die Schwierigkeiten beim Haushaltsbudget, führen zu einer Rezession, worauf es zu weiteren Zinserhöhungen und zu einer Abwertung der Währung kommt. Diese Automatik ist sehr schwer zu stoppen und vergrößert auf dramatische Weise die soziale Schieflage.

Die Situation vieler Länder ist heutzutage insofern paradox, als sie sich gleichzeitig verbessern und verschlechtern kann. Es findet zwar Wachstum statt, aber es ist ein langsameres und unstetiges Wachstum; die soziale Lage der Mehrheit der Bevölkerung ist instabil und innerhalb dieses Wachstumsregimes ist es unmöglich, die massive Armut nachhaltig zu bekämpfen.

Die Finanzlogik, die durch den Kasinokapitalismus eingeführt wurde, bringt eine erhebliche Instabilität des Wirtschaftslebens mit sich, entweder, weil Finanzkrisen vermieden werden sollen oder weil sie gerade ausgebrochen sind, wobei beide Erscheinungen miteinander verbunden sind. Wachstumsprofile sehen unter der Herrschaft dieser Finanzlogik aus wie zackige Gebirge. Insgesamt ist es ein Wachstum „auf Messers Schneide“.

Aus dem Französischen übersetzt von Dorothea Schmidt 


\section{Literatur}

Birdsall, N.; Londono J.L. (1997): Asset Inequality does matter : Lessons from Latin America. OCE Working Papers Inter American Bank of Development, Washington

Bourguignon, F (2000): Redistribution et développement. Conseil d'Analyse economique, La Documentation française. France

Calcagno, A.F. (2001): Ajuste estructural, costo social y modalidades de desarollo en America Latina, in: Sader E., El ajuste estructural en Americal Latina, costos sociales y alternativas ed. CLACSO et ASDI

CEPAL (2001): Una decada de luces y sombras, America Latina y el caraïbe en los anos noventa. in: CEPAL (Chili) et Alfaomega (Colombie) (Ed.): Panorama social de America Latina, Santiago du Chili.

CEPAL (2002): Globalzacion y desarrollo. Santiago du Chili.

CNUCED (1997): Trade and Development, Report 1997. Genève

De Janvry A.; Sadoulet, E. (1999): Growth, Poverty, and Inequality in Latin America, a causal Analysis, 1970-94. Inter-american Development Bank, Washington.

Destremau, B, et Salama, P (2001): De nouvelles causes au maintien de la paurreté, posface à l'édition. portugaise du livre des auteurs: Mesure et démesure de la pauvreté, ed Garamond, Brésil

Dias, David M (2001): Dynamique et permanence des exclusions au Brésil. Thèse Paris XIII, miméo, France

Fishlow, A (1996): Inequality, Poverty and Growth: where do we stand? Annual World Bank Conference on developement Economics, publié sous la direction de Bruno M et Plekovic B. World Bank.Washington

Jomo, K.S. (2001): Growth after the asian crisis: what remains of the east asian model? UNCTAD, G-24 Discussion paper $n^{\circ} 10$, Genève

Lustig, N. (1989): La desagualidad en Mexico, in: Economia de America Latina: las dimensiones sociales de la crisis, $\mathrm{n}^{\circ} 18 / 19$ CET Mexico

Katz, J. (2000): Reformas estructurales, productividad y conducta tecnologica en America Latina, Fondo de cultura economico et CEPAL; Pasado y presente del comportamiento tecnologico en America latina, document CEPAL, série desarollo economico $n^{\circ} 75$

Lustig, N. (2000): Crisis and the Poor; Socially Responsible Macroeconomics Inter-american Development Bank (I.A.D.B.), Technical Papers Series Washington

Marquez, G. (2000): Labor Markets and Income Support: What Did We Learn from the Crisis? Working Paper $n^{\circ} 425$, I.A.D.B., Washington.

Mathias, G.; Salama, P (1986): El estado sobredesarrollado. ed Era Mexique.

OIT (2000): Informa, America Latina y el Caraïbe, Panorama laboral '99. Suisse

Paes de Barros, R.; Mendonça, R. (1997): $O$ impacto do crescimento economico e de reduçoes no grau de desigualidade sobre a pobreza. texteo par discussao n528 IPEA

Paes de Barros, R.. et al. (2000): Poverty, Inequality and Macroeconomic Instability. Texto para discussao $n^{\circ} 750$, IPEA

Rodrik, D. (2000): Growth Versus Reduction: A Hollow Debate, in: Finance and devlopment vol.37, nº4 F.M.I. Washington

Salama, P. (1995): Pauvretés, les voies étroites d'une issue équitable en Amérique latine, in: Tiers Monde $n^{\circ} 142$

Salama, P. (1999): Riqueza e pobreza en america latina, la fragilidad de las nuevas politicas economicas. Ed Fondo de cultura economico, Mexique

Salama, P.; Valier, J. (1991): L'économie gangrénée: essai sur l'hyperinflation, ed La Découverte ;

UNCTAD (1997): Trade and Development, Report 1997, Genève.

UNCTAD (2002): Trade and Development, Report 2002, Genève.

Valier, J. (2000): Pauvreté, inégalités et politiques sociales dans les Tiers-Mondes depuis la fin des années quatre-vingt. Conseil d'Analyse economique, La Documentation française. France

Wodon, Q.T. (2000): Poverty and Policy in Latin America and the Carabean, Weltbank 